\title{
Surface-Enhanced Infrared Spectroscopy using ultra- compact indium tin oxide (ITO) sensor arrays
}

\author{
Yudong Wang ${ }^{12}$, Martina Abb ${ }^{2}$, Nikitas Papasimakis ${ }^{3}$, C. H. de Groot ${ }^{1}$, Otto L. Muskens ${ }^{2}$ \\ ${ }^{I}$ Nano Group and ${ }^{2}$ Physics \& Astronomy, Faculty of Physical Sciences and Engineering, and ${ }^{3}$ Optoelectronics Research Centre and Centre for \\ Photonic Metamaterials, University of Southampton, Highfield, Southampton SO17 1BJ, United Kingdom \\ Authore-mail address: ydw08r@ecs.soton.ac.uk; o.muskens@soton.ac.uk
}

\begin{abstract}
Reduced cross section and strong plasmon confinement allows ITO antennas to be integrated at extremely high densities with no loss in performance due to long-range transverse interactions and to hold promise for extremely sub-wavelength SEIRS.

OCIS codes: (250.5403) Plasmonics; (240.6680) Surface plasmons
\end{abstract}

\section{Introduction}

The large free carrier density and relatively small ohmic losses of the noble metals Au and Ag have enabled many of the breakthroughs in field-enhanced spectroscopy [1]. With the maturation of plasmonics research and its growing relevance for technology, new combinations of plasmonic materials with complementary functionalities are being sought, which could improve performance or could lead to entirely new application areas.

Alternative materials for plasmonics include a wide range of metals [2], doped semiconductors [3], metal oxides, nitrides [4], and graphene-like two-dimensional materials [5]. Next to conventional applications in the visible and near-infrared spectral range, some of these materials target the mid-infrared and terahertz domains with applications in, for example, chemical sensing and security.
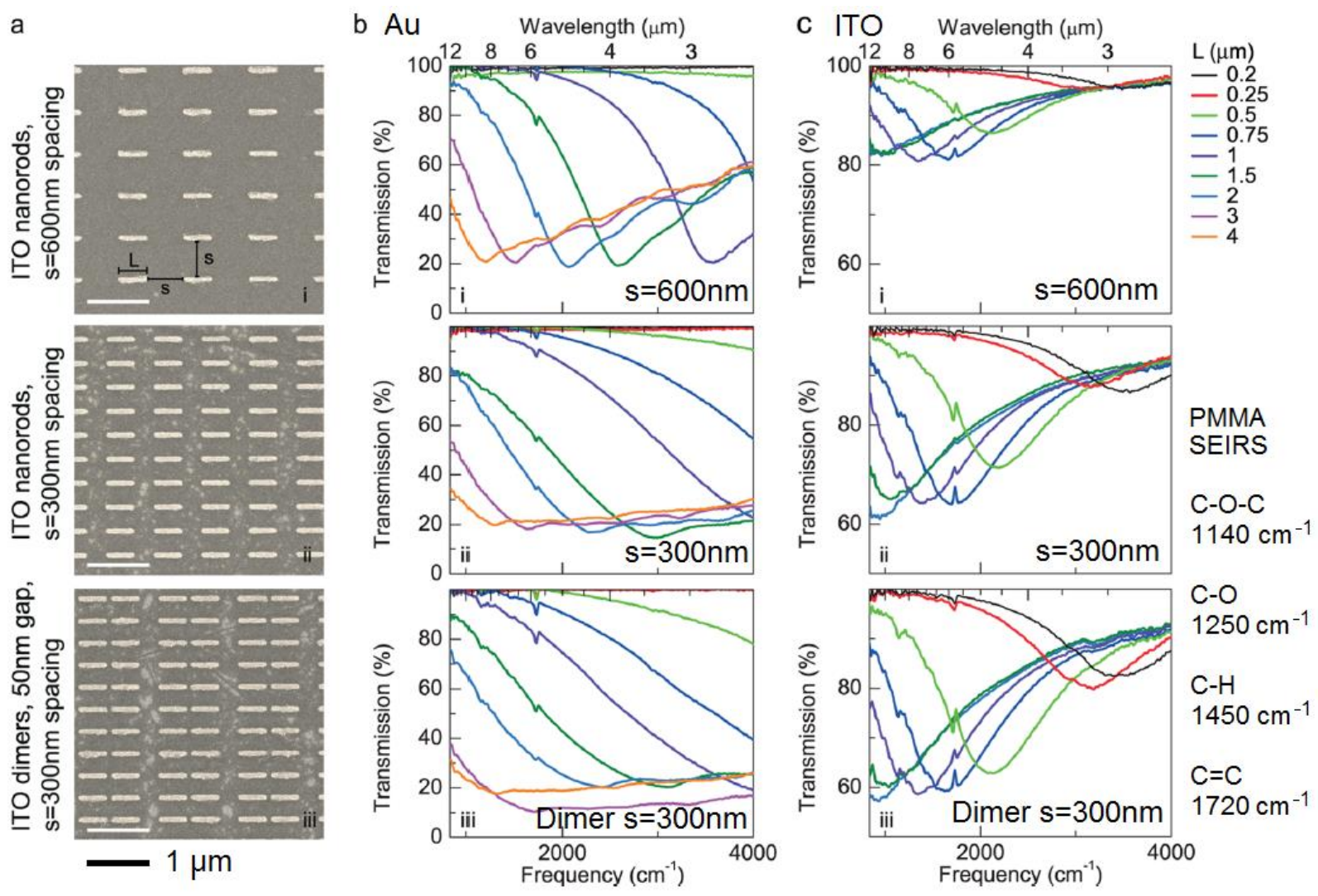

Fig. 1. (a) SEM images of ITO antenna arrays with $L=0.5 \mu \mathrm{m}$ for spacings $s=600 \mathrm{~nm}$ (i) and $s=300 \mathrm{~nm}$ (ii), and dimer antennas with $50 \mathrm{~nm}$ gap and $s=300 \mathrm{~nm}$ (iii). (b, c) Infrared transmission spectra of Au (b) and ITO (c) antenna arrays corresponding to typical array geometries presented in a and for various nanorod lengths L. The arrays were covered with a $50 \mathrm{~nm}$ PMMA layer (vibrational modes' frequency listed on the right hand side), resulting in sharp SEIRS features due to plasmon coupling with vibrational resonances. 


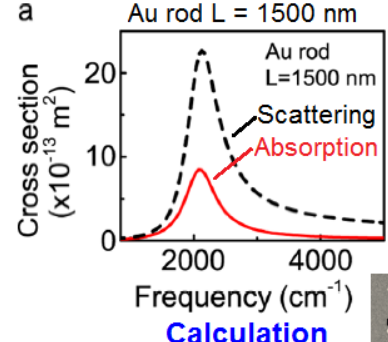

b

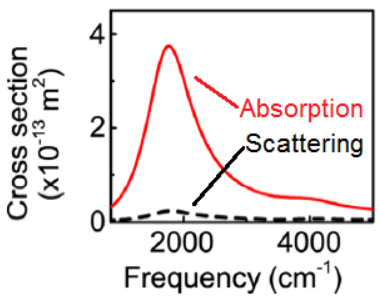

C

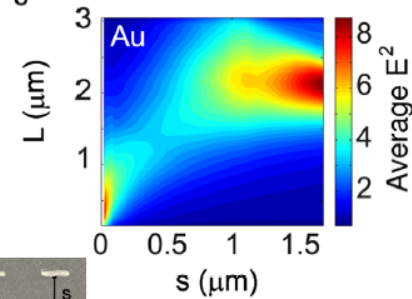

$\mathrm{s}(\mu \mathrm{m})$

Calculation

d

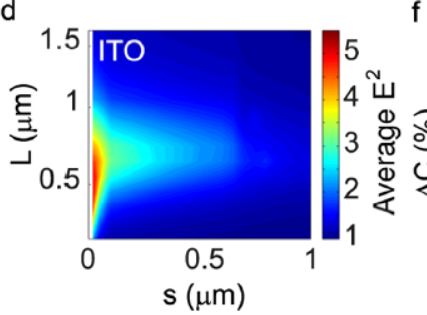

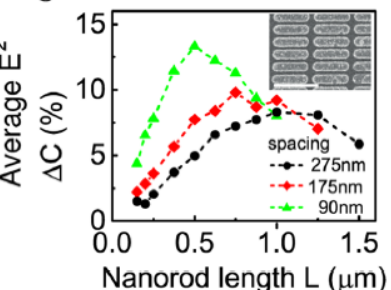

Experiment

f

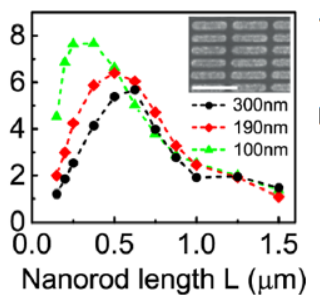

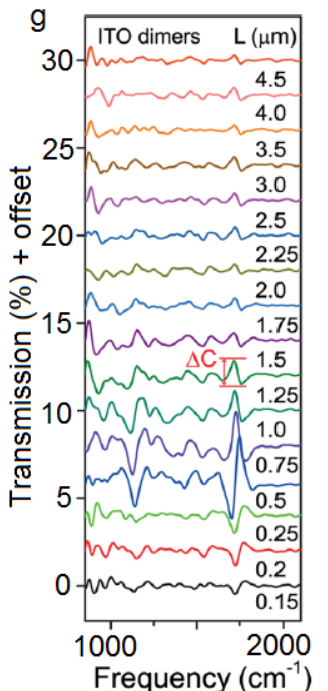

Fig. 2. (a, b) Calculated far-field absorption (solid line, red) and scattering (dashed line, black) cross sections for isolated $L=1500$ nm $\mathrm{Au}$ (a) and $L=750 \mathrm{~nm}$ ITO (b) rods on a $\mathrm{CaF}_{2}$ substrate. (c, d) Maps showing the average intensity enhancement over a plane at $20 \mathrm{~nm}$ above the substrate and at $1720 \mathrm{~cm}^{-1}(\mathrm{C}=\mathrm{C})$, for periodic arrays with spacings $s$ and antenna lengths $L$, for Au (c) and ITO (d). (e, f) Experimental fingerprint contrast for arrays with spacings between 90 and $300 \mathrm{~nm}$ for Au and ITO. (g) Vibrational spectra obtained using SEIRS, taken by subtracting 50pt smoothed curve from spectra of Figure 1c for dimer antennas iii. Arrow shows definition of fingerprint contrast $\triangle C$ of $\mathrm{C}=\mathrm{C}\left(1720 \mathrm{~cm}^{-1}\right)$.

\section{Experiment and Achievement}

Here we show that arrays of indium tin oxide (ITO) plasmonic nanoantennas (Figure 1) are highly suitable for surface-enhanced infrared spectroscopy (SEIRS) according to the measured infrared transmission spectra. SEIRS is a spectroscopic technique used to identify molecular fingerprints by resonant detection of infrared vibrational modes through coupling with the plasmonic modes of an antenna. Most SEIRS studies so far have used the noble metals $\mathrm{Au}$ and $\mathrm{Ag}$ as these have been shown to produce strong SEIRS signatures with monolayer sensitivity. Compared to $\mathrm{Au}$, the spectral resonance positions for ITO antenna arrays do not depend on antenna spacing $s$, which suggests that the ITO antennas are much less affected by interparticle coupling. [6]

The broad dips in the transmission spectra (Figure 1b,c) correspond to longitudinal surface plasmon resonances of the nanorods (Au, ITO). These resonances shift to lower energy with increasing antenna length. Compared to the $\mathrm{Au}$ antennas, the ITO antennas show infrared resonances for much shorter antenna lengths. Additionally, the transmission dips are less strong for the ITO than for $\mathrm{Au}$, indicating a reduced extinction per antenna.

From Figure 2a,b, it can be seen that while the absorption cross sections are similar for both rods, the scattering efficiency of the ITO is strongly reduced, which is in qualitative agreement with the analytical model for radiation damping. The near-field coupling is associated with a strong shift of the resonance to shorter antenna lengths $L$. The calculation for $\mathrm{Au}$ antennas (Figure 2c) shows that reducing the spacing from $\mathrm{s}=600 \mathrm{~nm}$ to $\mathrm{s}=300 \mathrm{~nm}$ results in lower enhancement, as observed in experiments. The ITO antennas (Figure 2d) show a monotonous increase of the resonant intensity enhancement with reduced spacing. Only for spacings $s$ below $300 \mathrm{~nm}$ is a spectral shift observed attributed to near-field coupling. Figures $2 \mathrm{e}$,f show that further reduction of the spacing results in an additional increase of the SEIRS enhancement and a shift of the resonance length.

\section{References}

[1] Moskovits, M. "Surface-enhanced spectroscopy" Rev. Mod. Phys. 1985 (57) 783-826.

[3] Knight, M. W.; Liu, L.; Wang, Y.; Brown, L.; Mukherjee, S.; King, N. S.; Everitt, H. O.; Nordlander, P.; Halas, N. J. "Aluminum Plasmonic Nanoantennas" Nano Lett. 2012 (12) 6000-6004.

[4] Law, S.; Yu, L.; Rosenberg, A.; Wasserman, D. "All-Semiconductor Plasmonic Nanoantennas for Infrared Sensing” Nano Lett. 2013 (13) $4569-4574$.

[5] Naik, G. V.; Shalaev, V. M.; Boltasseva, A. “Alternative Plasmonic Materials: Beyond Gold and Silver” Adv. Mater. 2013 (25) $3264-3294$.

[6] Chen, J.; Badioli, M.; Alonso-González, P.; Thongrattanasiri, S.; Huth, F.; Osmond, J.; Spasenović, M.; Centeno, A.; Pesquera, A.; Godignon, P.; Zurutuza Elorza, A.; Camara, N.; García de Abajo, F. J.; Hillenbrand, R.; Koppens, F. H. L. "Optical nano-imaging of gate-tunable graphene plasmons" Nature 2012 (487) 77-81.

[7] Abb, M.; Wang, Y.; Papasimakis, N.; de Groot, C. H.; Muskens, O. L. "Surface-Enhanced Infrared Spectroscopy Using Metal Oxide Plasmonic Antenna Arrays" Nano Lett. 2014 (14), 346-352. 